\title{
Electronic Structure Control of Sub-nanometer 1D SnTe via Nanostructuring within Single- Walled Carbon Nanotubes
}

Andrij Vasylenko, ${ }^{* \dagger \oplus}$ Samuel Marks, ${ }^{\dagger}$ Jamie M. Wynn, ${ }^{\ddagger}$ Paulo V. C. Medeiros, ${ }^{\ddagger \oplus 0}$ Quentin M. Ramasse, ${ }^{\S \odot 0}$ Andrew J. Morris, ${ }^{\perp \odot}$ Jeremy Sloan, ${ }^{*}, \dagger$ and David Quigley ${ }^{*}, \dagger$

${ }^{\dagger}$ Department of Physics, University of Warwick, Coventry, CV4 7AL, United Kingdom

${ }^{\ddagger}$ Cavendish Laboratory, University of Cambridge, Cambridge, CB3 OHE, United Kingdom

${ }^{\S}$ SuperSTEM Laboratory, SciTech Daresbury Campus, Daresbury, WA44AD, United Kingdom

${ }^{\perp}$ School of Metallurgy and Materials, University of Birmingham, Birmingham, B15 2TT, United Kingdom

Supporting Information

ABSTRACT: Nanostructuring, e.g., reduction of dimensionality in materials, offers a viable route toward regulation of materials electronic and hence functional properties. Here, we present the extreme case of nanostructuring, exploiting the capillarity of single-walled carbon nanotubes (SWCNTs) for the synthesis of the smallest possible SnTe nanowires with cross sections as thin as a single atom column. We demonstrate that by choosing the appropriate diameter of a template SWCNT, we can manipulate the structure of the quasi-one-dimensional (1D) SnTe to design electronic behavior. From first principles, we predict the structural re-formations that $\mathrm{SnTe}$ undergoes in varying

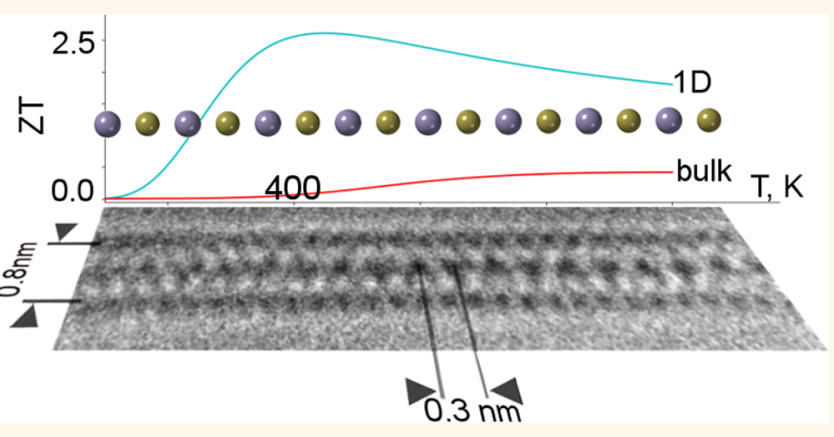
encapsulations and confront the prediction with TEM imagery. To further illustrate the control of physical properties by nanostructuring, we study the evolution of transport properties in a homologous series of models of synthesized and isolated SnTe nanowires varying only in morphology and atomic layer thickness. This extreme scaling is predicted to significantly enhance thermoelectric performance of $\mathrm{SnTe}$, offering a prospect for further experimental studies and future applications.

KEYWORDS: nanostructuring, nanowires, encapsulation, carbon nanotubes, crystal structure prediction, transport, thermoelectrics

$\mathrm{F}$ abrication of low-dimensional nanostructured materials has attracted much attention because of the extraordinary electrical and thermal properties the materials achieve at the nanoscale as well as their versatile applications in nanotechnology. Controllable synthesis of nanowires and fundamental investigation of their properties are essential for prospective applications including energy conversion ${ }^{1}$ and nanoelectronics. ${ }^{2}$ Regulation of the local structure at the nanoscale is of particular interest, as it enables the design of the properties and enhancement of the materials' performance for specific applications. In particular, optimization of thermoelectric efficiency can be achieved due to band structure engineering and reduction of lattice thermal conductivity or scattering engineering. ${ }^{3-12}$ Scaling to the lower dimensions leads to synergy of these strategies for enhancement of thermoelectric performance; ${ }^{13-15}$ however, up until recently this remained practically unachievable for many of the most promising candidate materials. ${ }^{1}$ Hence development of techniques for controlled fabrication of nanowires with a potential to regulate local structure is necessary for improvement of the current level of thermoelectric efficiency.

A candidate material, tin telluride, has a moderate thermoelectric efficiency due to characteristic high thermal conductivity and subsequently low thermoelectric figure of merit (ZT) in its pure bulk crystalline form. ${ }^{16-18}$ However, the accessible bond hybridization and phase change properties of $\mathrm{SnTe}^{19,20}$ imply rich diversity in local structure, ${ }^{16-18}$ possibly leading to dramatically different structures under extreme confinement. ${ }^{21-23}$ Single-walled carbon nanotubes (SWCNTs) with diameters ranging from 7 to $14 \AA$ can be used as templates with a controllable degree of confinement for the growth of quasi-one-dimensional (1D) nanocrystals. ${ }^{21,22,24-27}$ Templating or encapsulating materials inside SWCNTs as a function of

Received: March 26, 2018

Accepted: May 21, 2018

Published: May 21, 2018 

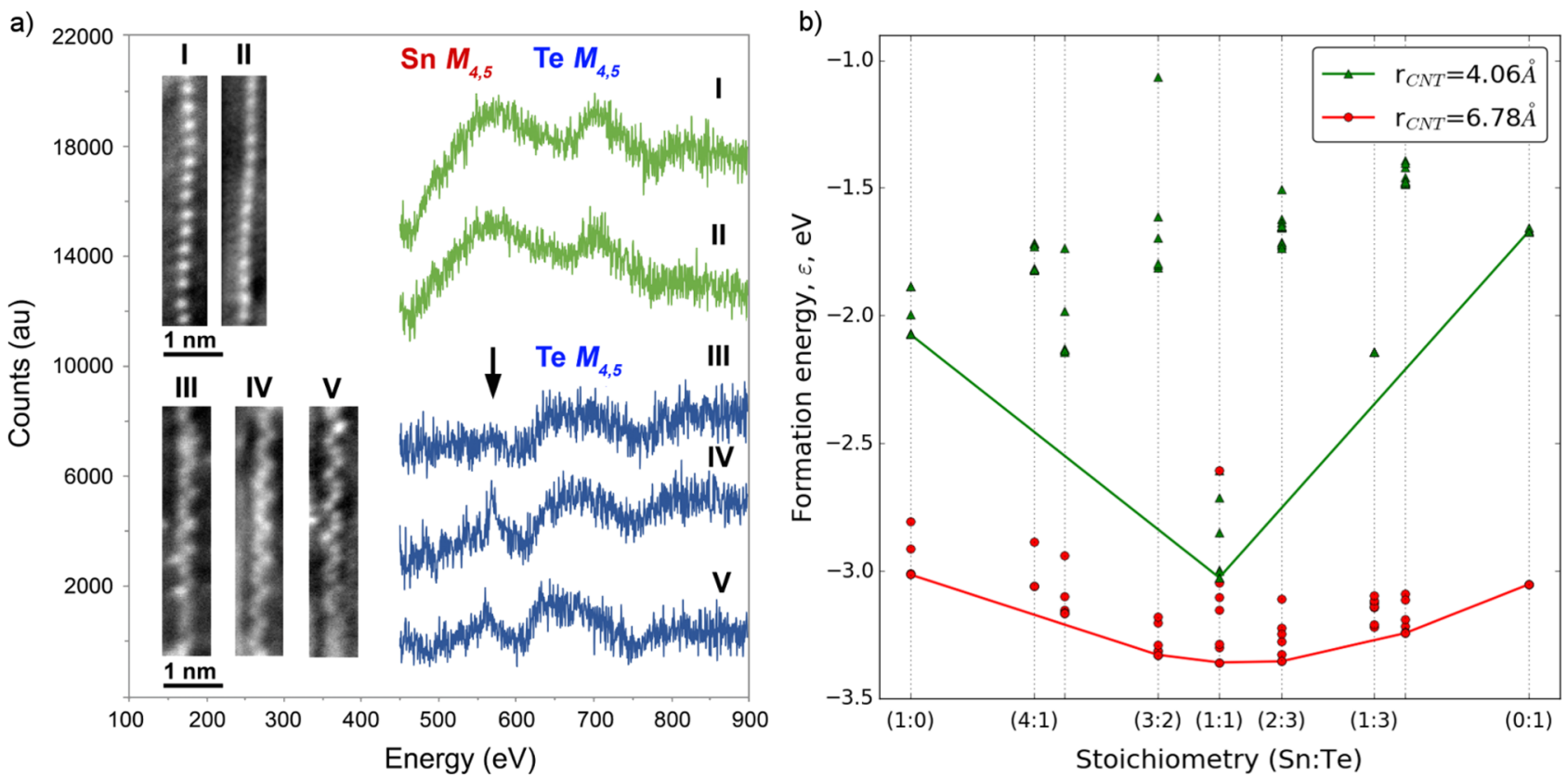

Figure 1. (a) EELS at the outmost electron shells (M-edge) in SnTe 1D atomic chains and Te coils encapsulated within SWCNTs. Note that Te coils are only obtained in nanowires depleted in $\mathrm{Sn}^{24}$ as indicated with an arrow. The small difference in atomic number for Sn $(\mathrm{Z}=50)$ and $\mathrm{Te}(\mathrm{Z}=52)$ results in highly similar contrast for these atoms in the observed SnTe experimental images and simulations. However, the summed EELS spectra from images I and II (i.e., obtained from the same field of view in both instance) show a clear difference in both composition and linear (or zigzag) morphology than from the images and summed EELS spectra from images III to V, the latter showing a coil-like morphology only, consistent with the results we reported for pure Te "coils" in ref 24 . (b) Convex hull construction for SnTe nanowires with different stoichiometries embedded in $\operatorname{CNT}(6,6), r=4.06 \AA$ and $\operatorname{CNT}(10,10), r=6.78 \AA$. The formation energy per atom is plotted for chemical potentials of $\mathrm{Sn}$ and Te relevant to an ideal gas 1:1 mixture with a density of $5.00 \mathrm{~g} / \mathrm{L}$ at $300 \mathrm{~K}$. In CNTs with small radius (green line) only pure $\mathrm{Sn}$, pure $\mathrm{Te}$, or $\mathrm{Sn}_{1} \mathrm{Te}_{1}$ nanowires can be formed, with $\mathrm{SnTe}$ being the most energetically favorable composition for infinitely long nanowires. In CNTs with larger diameters (red line) composition selectivity is weaker, such that in small regions disproportionation into 2:3 and 3:2 stoichiometry is energetically competitive. Triangles and circles represent 100 of the lowest formation energies of the structures predicted with AIRSS.

their van der Waals internal surface has been suggested as far back as 1997, when Nikolaev et al. ${ }^{28}$ indicated that $\mathrm{C}_{60}$ was just the right diameter to fit in $1.4 \mathrm{~nm}$ SWCNTs, subsequently realized by Smith et al. ${ }^{29}$ We and many others have investigated the "integral atomic layer" growth of metal halides in SWCNTs in terms of their van der Waals confinement, ${ }^{30,31}$ and many other studies since have explored the confinement and transport of many other species including, for example, ions, ${ }^{32}$ graphene ribbons, ${ }^{33}$ and, in the narrowest nanotubes, single atomic chains of silicon. ${ }^{34}$ Senga et al. ${ }^{26}$ described the subsequent formation of CsI in DWCNTs, and we recently reported $\mathrm{Te}$ atomic chains and coils in the narrowest SWCNTs; ${ }^{24}$ much of this work has been done with a view to either tailoring the physical properties of the confining materials or modifying the properties of the included materials by confinement. Due to the often unexpected $1 \mathrm{D}$ structures that phase change materials may manifest in extreme confinement, one cannot deduce their crystallography on the basis of their known bulk counterparts. ${ }^{24}$ Reduced dimensionality, in particular, gives rise to surface effects in electronic transport and multiple phonon-drag enhancement of thermoelectric properties. ${ }^{1,15,35,36}$ Understanding the effect of confinement on the structure and transport properties can thus enable development of a powerful tool for the regulation of thermoelectric performance in such materials.

\section{RESULTS AND DISCUSSION}

Here we study a controlled synthesis of the smallest possible 1D crystalline SnTe nanowires within SWCNTs and the change in their structure and electronic behavior as a function of varying degrees of confinement. To predict the structure and stoichiometry of SnTe nanowires encapsulated in SWCNTs, we employ $a b$ initio random structure searching (AIRSS) ${ }^{37}$ analyzed via a convex hull construction. ${ }^{38,39}$ This allows for visualization of formation energy for a range of available structures and stoichiometries. We then calculate energy of formation from gaseous $\mathrm{Sn}$ and $\mathrm{Te}$ and determine the thermodynamic stability of $\mathrm{SnTe}$ nanowire structures within SWCNTs of different diameters. During synthesis, we apply a sublimation technique for the growth of $\mathrm{SnTe}$ within SWCNTs. ${ }^{26}$ We then employ aberration-corrected high resolution transmission electron microscopy (HRTEM), aberration-corrected scanning transmission electron microscopy (STEM), and electron energy loss spectroscopy (EELS) to experimentally verify the structures obtained, hence demonstrating we have proposed a viable synthesis route.

To study the encapsulated SnTe nanocrystals, we first identify the most stable structures adopted by this material when confined within SWCNTs. We previously adapted AIRSS for the prediction of $1 \mathrm{D}$ structures that manifest within SWCNTs of various diameters. ${ }^{24}$ In the present work by applying the same strategy, i.e., by optimizing geometries of random structures in confinement of explicit CNTs or confining potential, we generate more than 3100 structures 

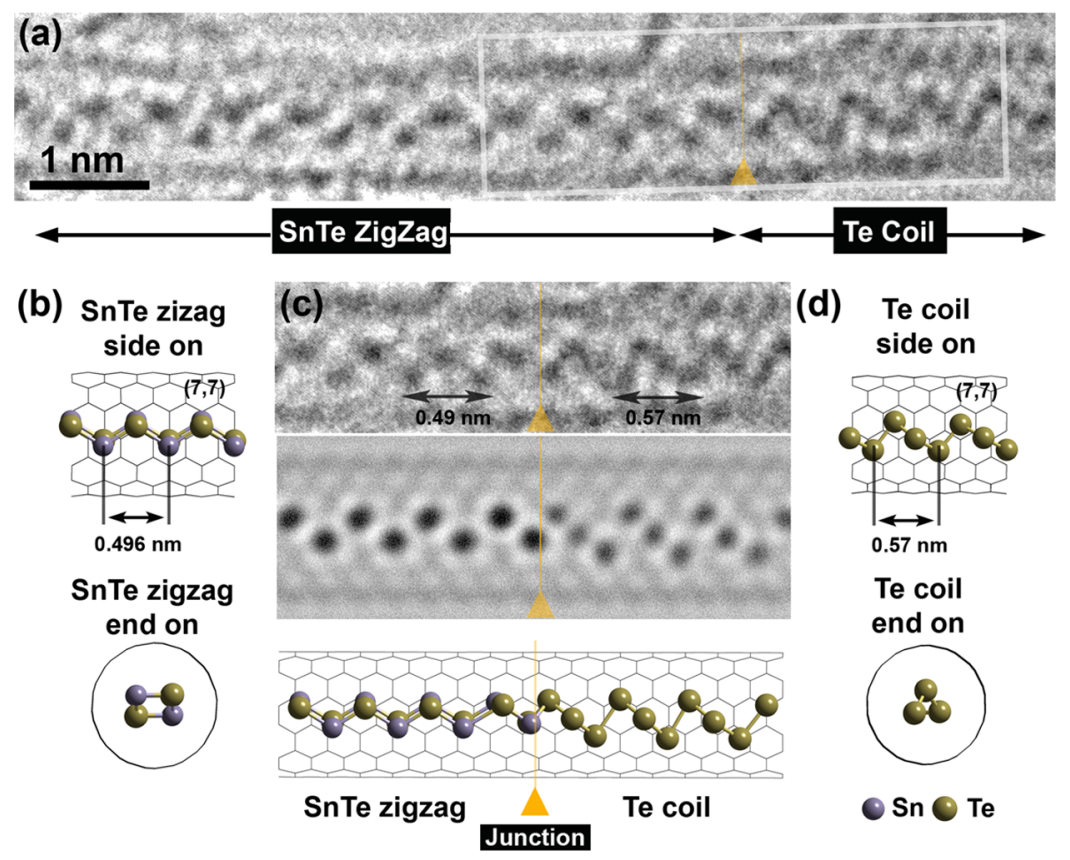

Figure 2. A $\sim 8 \mathrm{~nm}$ section of a $0.95 \mathrm{~nm}$ diameter SWNT containing two distinct crystalline regions: a SnTe double zigzag and a Te coil region depleted in Sn (cf. Figure 1(a), Figure 4, and ref 1). (b) Schematic side-on and end-on views of double zigzag SnTe in a (7,7) SWCNT. (c) Detail from (a), multislice TEM simulation, and structure model of the SnTe zigzag: Te coil junction. (d) Schematic side- and end-on views of a Te coil in a $(7,7)$ SWCNT $(c f$. ref 1$)$.

with different stoichiometries, encapsulated within a variety of radii of CNTs. In order to exclude artificial strain in modeling of the systems with incommensurate sublattices, for calculations of lattice and energy corrections we exploit a theoretical construct of an implicit nanotube: a cylindrical electrostatic potential that provides steric confinement of SnTe. The technicalities and justification of such an approach are detailed in ref 24. Sampling of the stoichiometries is important due to potential disproportionation of the $\mathrm{Sn}$ :Te ratio during sublimation of the solid. In the convex hull construction, formation energy $\varepsilon$ of the randomly generated structures upon $a b$ initio optimization of their geometries is plotted against stoichiometry. In this case per-atom formation energy is calculated with respect to the chemical potentials in a reservoir of ideal gas of $\mathrm{Sn}$ and Te atoms, i.e., which are produced via sublimation of $\mathrm{SnTe}$ under typical experimental conditions.

$$
\varepsilon=\frac{E_{\text {total }}-E_{\mathrm{CNT}}}{N}-\mu_{\mathrm{Sn}}-X_{\mathrm{Te}}\left(\mu_{\mathrm{Te}}-\mu_{\mathrm{Sn}}\right)
$$

where $E_{\text {total }}$ is the total energy of a nanowire encapsulated within SWCNTs, $E_{\mathrm{CNT}}$ is the energy of the corresponding pristine SWCNTs, $N$ is the number of atoms in the encapsulated structure, $\mu$ stands for the chemical potential of the corresponding species, and $X_{\mathrm{Te}}=N_{\mathrm{Te}} / N$ is the fraction of $\mathrm{Te}$ atoms within the encapsulated structure. Thermodynamically stable structures for a given stoichiometry form the convex hull of points, with the lowest energy vertex identifying the most energetically favorable composition for an infinitely long nanowire (Figure $1 \mathrm{~b}$ ). These diagrams reveal a selectivity for nanowires with equal proportion of $\mathrm{Sn}$ :Te within the smallest SWCNTs (i.e., green lines in Figure 1b). This finding is indirectly confirmed by the experimentally obtained EELS that features peaks from $\mathrm{Sn}$ and Te atoms with equal intensity for the single-atom chains formed within the smallest SWCNTs ((I) and (II) in Figure 1a). In SWCNTs with larger diameters (red lines in Figure 1b), the difference between the formation energy of the encapsulated nanowires decreases, and compounds with varying stoichiometry other than a $1: 1$ ratio become more competitive. Thus, some finite size regions of the synthesized samples within larger diameter SWCNTs are expected to be depleted of, or enriched in, Sn. In our experimental findings, we are able to identify a region of the sample where SnTe with a 1:1 stoichiometry is present within the same nanotube as helical $\mathrm{Te}^{24}$ (see (III)-(V) in Figure 1a). This unexpected behavior suggests that the kinetics of filling SWCNTs from the gas phase may lead to inhomogeneities in the encapsulated structures, leading to formation of junctions between $\mathrm{SnTe}$ and $\mathrm{Te}$ nanowires (see Figure 2). These two nanowire structures as predicted via AIRSS have two distinctively different motifs and lattice parameters, commensurate with those in the HRTEM image. Moreover, geometry optimization using first-principles indicates that a repeating periodic realization of this junction occupies a local minimum on the potential energy landscape. A model in which SnTe is forced to form a corresponding helical chain demonstrates dynamical instability and undergoes reconstruction into a double-zigzag structure during minimization of forces. The majority of encapsulated $\mathrm{SnTe}$ nanowires exhibit 1:1 stoichiometry at this CNT diameter.

After identifying the lowest energy nanowire structures via our AIRSS searches, we proceed to studying their relative stability as a function of degree of confinement within CNTs. By comparing the formation energy of the predicted structures within different diameters of SWCNTs, we have established a strong dependence of the structure formed on the diameter of the encapsulating SWCNTs. Via a series of detailed calculations (see Supporting Information (SI) for the details of methods and calculations) we are able to plot the formation energy of each nanowire as a function of the confining SWCNT diameter and, hence, identify diameters at which particular structures are 
unambiguously stable. The results are shown in Figure 3. The formation energies of all of the investigated structures

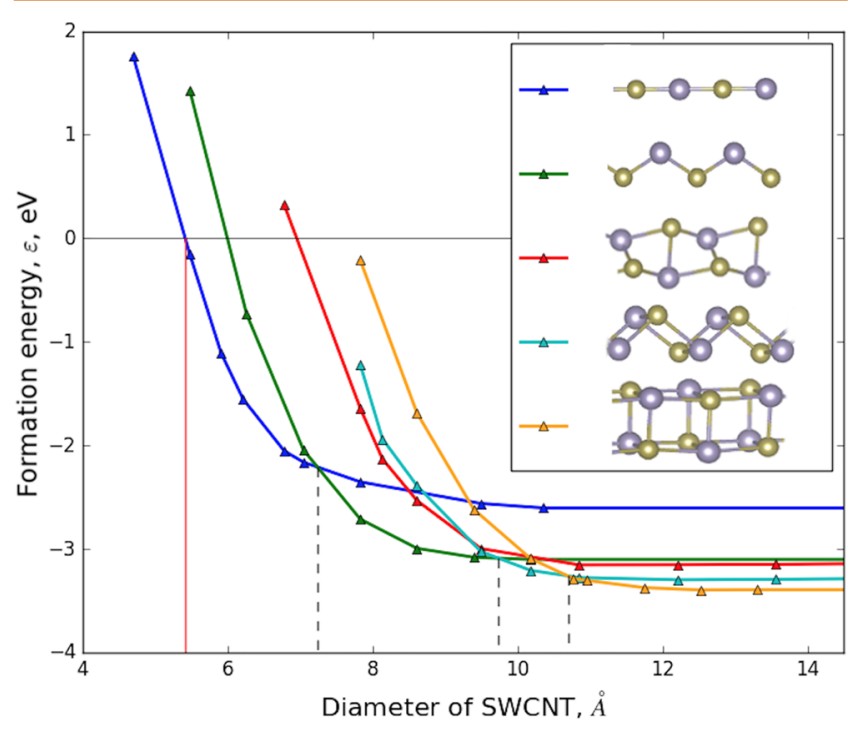

Figure 3. Evolution of the lowest energy structure as a function of encapsulating SWCNT diameter. Vertical lines indicate the degree of confinement, where formation of SnTe from gas is energetically accessible within a SWCNT (red); structural transitions occur (dashed).

demonstrate the same trend as the diameters of the encapsulating SWCNT increases: first a rapid drop followed by a leveling off at the value corresponding to noninteracting nanowire-SWCNTs. We also find a lower limit of $5.6 \AA$ for the smallest diameters of the encapsulating SWCNTs that can be filled with sublimed SnTe (vertical red line in Figure 3). In SWCNTs of certain larger diameters (black dashed lines in Figure 3) formation of different structures becomes energetically competitive, and hence transitions may occur between competing nanowire structures. The evolution of the nanostructure adopted by SnTe within SWCNTs with different diameters is presented in Figure 4, where we compare the structures predicted by AIRSS to those experimentally synthesized via simulated HRTEM images. We investigate the mechanical stability of each encapsulated nanowire structure in a series of $a b$ initio calculations of their lattice vibrational characteristics (see the SI for the details of phonon dispersions in the SnTe nanowires). From the latter, we find that in confinement in the regions between the transition points (black dashed lines in Figure 3), the corresponding lowest energy structures are mechanically stable. A polar linear chain of SnTe while being the most stable structure in the small-diameter CNTs undergoes reconstruction into a zigzag nanowire upon isolation from the CNTs. Phonon dispersions in the isolated zigzag, double-zigzag, and rocksalt-like nanowires retain the stability features, hence indicating the possibility for their isolation from CNTs. The role of encapsulating SWCNTs in stabilization and electronic interaction with the filling is further studied with Bader charge analysis, which does not demonstrate any substantial charge transfer (see the SI for the details of Bader charge analysis in the SnTe@SWCNTs compound systems). With chemical interaction between CNTs and encapsulated nanowires being negligibly small, the main interaction between the subsystems in this compound material is via steric confinement. We thus conclude that an appropriate choice of the host SWCNTs can allow controlled structuring of nanowires with increasing coordination numbers, from $1 \mathrm{D}$ atomic chains toward bulk-like rocksalt nanowires. Further extraction of the synthesized nanowires is possible due to the techniques for unravelling SWCNTs. ${ }^{42,43}$ Alternatively, the technique for synthesis of nanowires within CNTs performed in this work can be applied to carbon nanoscrolls of appropriate diameter with their subsequent unrolling. ${ }^{44,45}$

Electronic band structure engineering, in particular opening of the band gap and convergence of conduction bands, has been demonstrated as a valid route toward improvement of thermoelectric properties. ${ }^{7,8,12,18}$ Both approaches lead toward significant enhancement of the Seebeck coefficient and electron transport in nanostructured materials. We investigate regulation of the electronic structure in SnTe nanowires via synthesis within different diameter SWCNTs. We perform a series of calculations of the $k$-resolved PDOS and evaluate the partial distribution of electrons (via Lowdin charge distribution) during structural evolution of the nanowires (i.e., following the evolution of nanowire structure and electronic structure in concert with the indicated arrows in Figure 5). With an increase in the diameter of confinement, the atomic bond lengths and angles change, triggering rehybridization of the atomic orbitals and redistribution of the charge on them. On the outermost $5 \mathrm{~d}$ orbitals of both $\mathrm{Sn}$ and Te, there is a growth of the number of electrons participating in transport. Combined with the increase in the total number of electrons in the larger nanowires as well as the increased number of conduction bands in the vicinity of the Fermi level, the convolution of these factors results in a boost in electronic transport in a rocksalt-like nanowire (see Figure 5a). From first principles, we calculate the electronic band structure and corresponding effective electron mass $\frac{1}{m^{*}{ }_{\nu}}=\frac{1}{\hbar^{2}} \frac{\mathrm{d}^{2} E_{\nu}}{\mathrm{d} k^{2}}$ in the model lowest energy nanowires that can be synthesized within different diameter SWCNTs. Our calculations indicate that as the diameter of the confining SWCNT increases, the electronic band gap of the corresponding nanowire widens and the effective mass of carriers also increases; hence all of the studied nanowires except for the $1 \mathrm{D}$ linear chain exhibit semiconducting characteristics (see Figure 6).

Moreover, for the rocksalt-like nanowire that can be formed due to encapsulation inside SWCNTs with diameters $>11.2 \AA$, there is conduction and valence band convergence at the high symmetry points close to the Fermi level, leading to strongly enhanced transport properties (see Figure 5). The Fermi surface in all of the nanowires does not exhibit topological anisotropy due to the extreme scale. To demonstrate the profound effect that a proposed method of engineering of electronic band structures might have on the functional properties, we further conduct a series of $a b$ initio calculations of electron-phonon $\frac{1}{\tau_{\mathrm{el}-\mathrm{ph}}^{\nu}} \sim \sum_{\mathbf{k}, \mathbf{q}}\left|\left\langle\mathbf{k}\left|\frac{\partial^{2} E}{\partial \mathbf{k}^{2}}\right| \mathbf{k}+\mathbf{q}\right\rangle\right|^{2}$ and phonon-phonon scattering $\frac{1}{\tau_{\mathrm{ph}-\mathrm{ph}}^{\nu}} \sim \sum_{\mathbf{q}_{0^{\prime}} \mathbf{q},-\mathbf{q}}\left|\frac{\partial^{3} E}{\partial \mathbf{q}_{0} \partial \mathbf{q} \partial-\mathbf{q}}\right|^{2}$ and the ultimate applications, in which $\mathrm{SnTe}$ nanowires are isolated from the templates for synthesis, evaluate transport properties by solving the corresponding Boltzmann transport equations for electrons and phonons. We validate this $a b$ initio-based methodology for evaluation of transport characteristics by calculating ZT for bulk SnTe crystal, for which our results are in close agreement in functional trend and minimum and maximum values with a previous experiment (see red dashed 


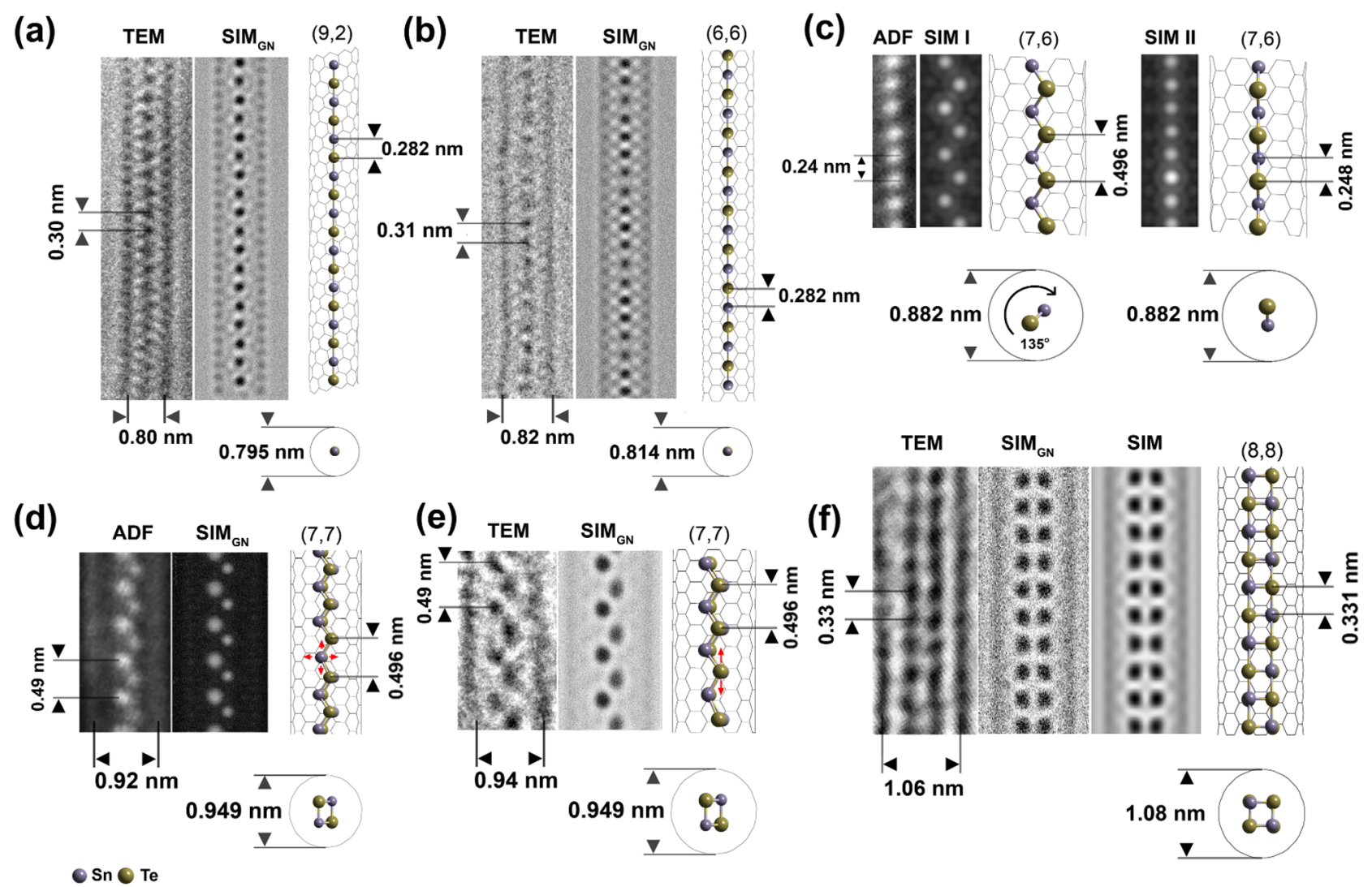

Figure 4. Evolution of the SnTe nanowire within SWCNTs of increasing diameters from a single-atom chain (a, b) to a zigzag chain viewed in plan view as indicated by the models and simulations (c) to a double-zigzag chain (d, e) and then a $2 \times 2$ rocksalt-like SnTe fragment (f). Some distortions, including asymmetric distortions, are evident in the experimental images in (d), (e), and (f) probably caused by thermolytic interactions between the electron beam and the specimen similar to those reported for $\mathrm{SnSe}{ }^{22}$ To reproduce these effects in the simulations, we have introduced atom column superposition and/or staggering with added "virtual" atoms to reproduce the blur in two directions, as indicated by the small red arrows in the corresponding models on the LHS atoms (d) or the RHS atoms (e) (key: TEM = $80 \mathrm{kV}$ aberrationcorrected TEM image; $\mathrm{SIM}_{\mathrm{GN}}=$ multislice TEM or ADF image simulation with $15 \%$ Gaussian noise added; ADF $=$ aberration-corrected annular dark field STEM image; SIM = multislice ADF image simulation (no noise); SWCNT conformations indicated by integers in parentheses).

line vs black circle line in Figure 6). We find that the Seebeck coefficient for the SnTe 1D chain, the double-zigzag SnTe chain, and the $2 \times 2 \mathrm{SnTe}$ rocksalt chain nanowires are all significantly enhanced in comparison either to the bulk or to bulk-doped SnTe. ${ }^{40,41}$ This enhancement coupled with high electrical conductivity results in an ultrahigh boost of the power factor, up to $1.9 \mathrm{~W} /\left(\mathrm{K}^{2} \cdot \mathrm{cm}\right)$ at $1000 \mathrm{~K}$. Decrease of thermal conductivity, $\kappa$, is another important target that can often be effectively addressed by reduction in dimensionality. ${ }^{13,35}$ Due to the surface-dominated phonon scattering, nanowires of small diameters demonstrate a significant decrease in phonon lifetimes and thus limited lattice thermal conductivity. ${ }^{13,35}$

We have previously demonstrated that in nanocomposite structures SWCNT-nanowire phonon lifetimes can be further decreased due to the extra pathways for phonon decay. ${ }^{46}$ Further research is required to investigate how this affects lattice thermal conductivity of nanocomposite SWCNTnanowire, and SnTe in particular. In the case where encapsulation of nanowires induces phonon modes so that more phonons satisfy the selection rules, we can expect effective redistribution of the phonon population, which may decrease surface phonon conductivity. ${ }^{47}$ In such compound nanostructures, with electronic and thermal transport dominated by CNTs, reduction in lattice thermal conductivity would lead to improved thermoelectric performance in comparison to that of pristine CNTs. This renders nanowires encapsulated in CNTs as a novel prospect in highly effective thermoelectric materials.

Here, we show that total thermal conductivity in the smallest isolated $\mathrm{SnTe}$ nanowires is dominated by the colossal electron contribution to heat transport and reaches $9.8 \mathrm{~W} /(\mathrm{K} \cdot \mathrm{m})$ at $1000 \mathrm{~K}$ for the rocksalt-like nanowire (see SI for the details of calculations of transport properties in the SnTe nanowires). Due to the moderate steepness of the trend, however, $\kappa$ does not exceed $2 \mathrm{~W} /(\mathrm{K} \cdot \mathrm{m})$ at temperatures below $600 \mathrm{~K}$. As with the Seebeck coefficient, the resulting overall ZT for SnTe nanowires that can be synthesized within SWCNTs is significantly enhanced in comparison to previously realized figures for bulk doped. ${ }^{41}$ This is achieved over a wide temperature range, reaching 3.25 at $380 \mathrm{~K}$ for the rocksaltlike nanowire (see Figure 6).

\section{CONCLUSIONS}

We have demonstrated that the confinement provided by SWCNTs with diameters from 7 to $11 \AA ̊$ can be exploited as a viable route for synthesis of the smallest possible SnTe 1D nanowires with a tunable electronic behavior. We discovered several possible, previously unreported, structural configurations of the atomic scale nanowires that are energetically 

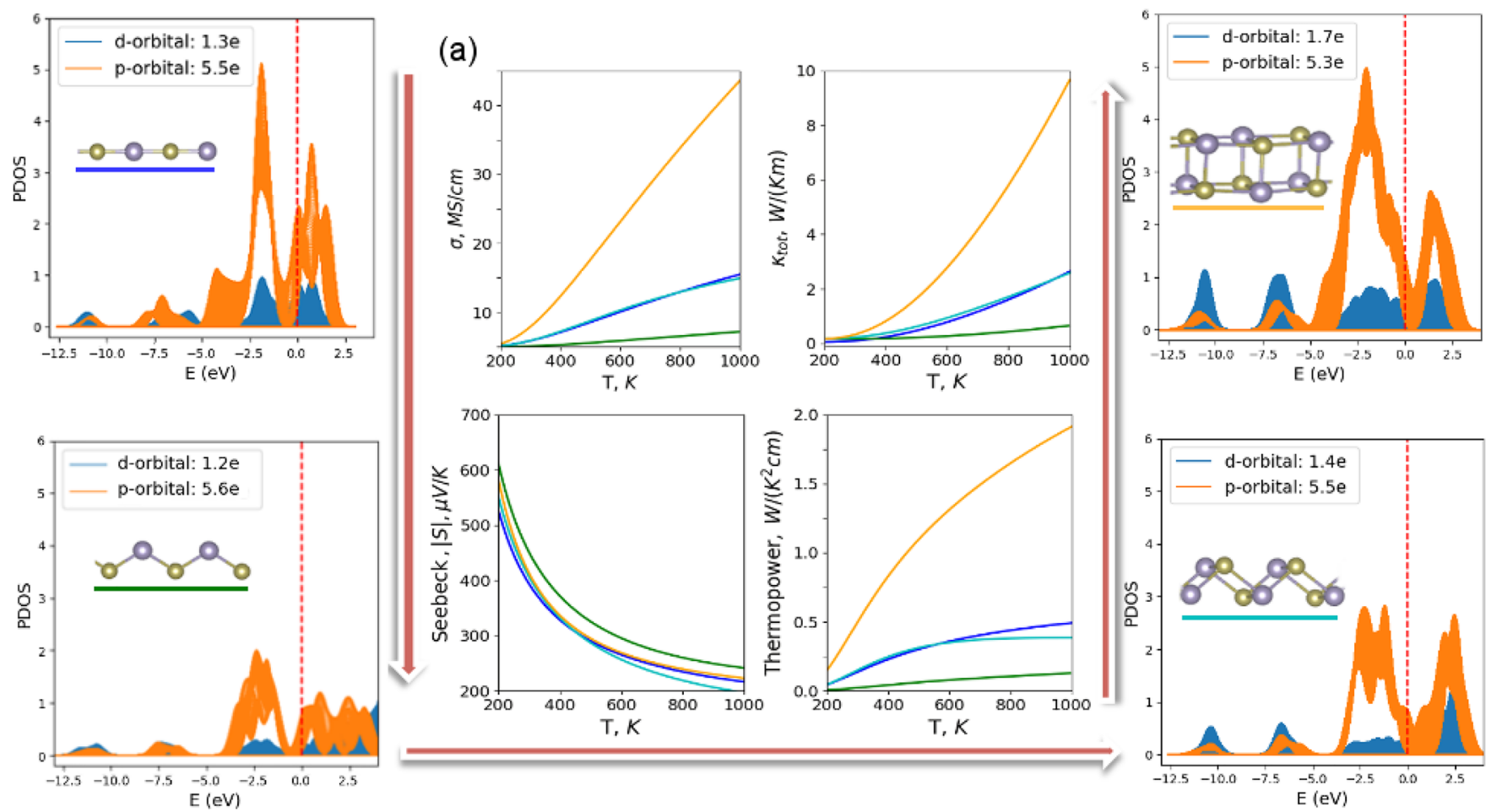

Figure 5. Electronic population on the outermost $p$ - and d-orbitals of $\mathrm{Sn}$ and Te and the partial density of states (PDOS) in encapsulated nanowires within SWCNTs of increasing diameter (in the direction of the arrow). Resolution PDOS in the reciprocal space is represented by the width of the curves. Rehybridization of atomic orbitals during structural evolution results in more electrons reaching 5d-orbitals. Due to the combination of the introduced transformations-increase of PDOS at the top valence level, more bands crossing the Fermi level (dashed line), and more electrons engaged as carriers-transport properties are enhanced (a).

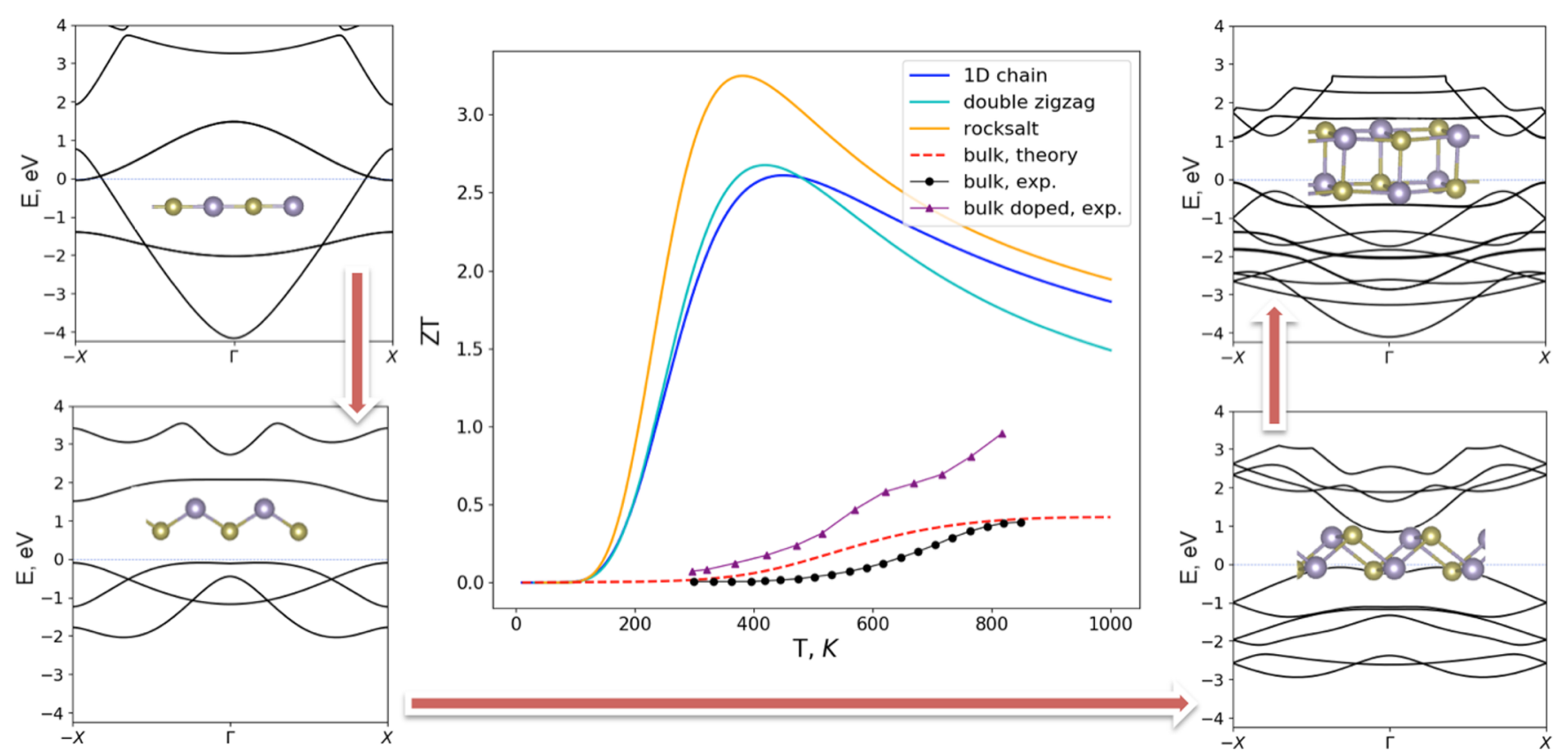

Figure 6. Evolution of electronic band structure, opening of the band gap, and ZT of the corresponding stable SnTe nanowires synthesized in SWCNTs of increasing diameter (in the direction of the arrow). The ZT value for the zigzag structure (green line in Figure 3) does not exceed 0.01 and is omitted for clarity. ${ }^{40}$ Experimental data for ZT of the bulk SnTe are taken from ref 18 . Experimental data for ZT of the bulk Cddoped $\mathrm{SnCd}_{0.03} \mathrm{Te}$ are taken from ref 41 .

preferable within SWCNTs of different diameters. We have determined that electronic structures of the formed nanocrystals depend directly on the diameters of the encapsulating SWCNTs used in synthesis, thus offering a practical tool for band structure engineering and, consequently, high thermoelectric performance via appropriate choice of templates for synthesis. Exploitation of homogeneous samples of SWNTs with diameters $>1.1 \mathrm{~nm}$ will enable controlled synthesis of 
rocksalt-like SnTe nanowires with the highest ZT. While the focus of this study was on the smallest $1 \mathrm{D}$ nanowires starting from the bottom up, notably a similar trend of increasing electron band gap and effective mass as in Figure 6 has been observed with the decrease of the diameter of the nanowires from $\sim 30 \mathrm{~nm}^{1}{ }^{1}$ This indicates the possibility for the existence of an optimal geometrical parameter (diameter) of the nanowires for which thermoelectric performance may reach its maximum value. With the models of SnTe nanowires isolated upon synthesis in SWCNTs exhibiting unprecedented high thermoelectric efficiency, previous reports on high ZT of CNTs themselves $^{48,49}$ inspire a hypothesis of overall high thermoelectric performance in a compound material. Verification of thermoelectric qualities of a compound material is increasingly possible upon formation of yarns or thin films of SnTe@ SWCNTs and warrants future experimental studies and applications.

\section{METHODS}

Sample Preparation. SWeNT SWNTs $(0.7-1.1 \mathrm{~nm})$ are supplied in the UK by Sigma-Aldrich but were originally manufactured by SouthWest NanoTechnologies as a specific grade (i.e., SWeNT SG65i, Product No. 773735) processed SWNTs produced by the CoMoCAT synthetic protocol. A $30 \mathrm{mg}$ amount of SWeNT CNTs was oxidized in the atmosphere at $750 \mathrm{~K}$ for $40 \mathrm{~min}$, reducing to $10 \mathrm{mg}$. A $70 \mathrm{mg}$ amount of SnTe (Sigma-Aldritch, 99.999\%) was loaded into the sealed end of a silica quartz sublimation ampule with the reduced CNTs in the other. The ampule was sealed under argon and heated to $1033 \mathrm{~K}$ for 7 days. The $\mathrm{SnTe}$ end of the ampule was placed in the center of the furnace, creating a negative temperature gradient toward the CNTs. Approximately $2 \mathrm{mg}$ of SnTe@SWCNTs was dispersed into $20 \mathrm{~mL}$ of 2-propanol and sonicated with pulsing (i.e. $2 \mathrm{~s}$ on / $2 \mathrm{~s}$ off) with a sonic probe for $40 \mathrm{~min}$. Three drops of dispersed CNTs were drop cast onto a $3.05 \mathrm{~mm}$ copper grid with a lacey carbon support film (Agar Scientific).

Electron Microscopy. The samples were examined in a JEOL double-aberration-corrected ARM200F electron microscope operating at $80 \mathrm{kV}$. Data were recorded on a Gatan Orius SC1000 $1.024 \times 1024$ pixel CCD camera. The STEM-EELS data were acquired on a Nion UltraSTEM100 instrument, operated at $60 \mathrm{kV}$ acceleration voltage and equipped with a cold field emission gun providing a native beam energy width of $0.30 \mathrm{eV}$. The optics were configured to form a $1.1 \AA$ probe (full-width at half-maximum) of $32 \mathrm{mrad}$ convergence semiangle, with a $40 \mathrm{pA}$ beam current. High- and medium-angle annular dark-field images were recorded with semiangular ranges of 85-190 and 55-80 mrad, respectively. EELS data were collected with a Gatan Enfina spectrometer with a collection semiangle of $36 \mathrm{mrad}$. A dispersion of $0.5 \mathrm{eV} /$ channel was chosen in order to record simultaneously the $\mathrm{C} \mathrm{K} \mathrm{SnM}{ }_{4,5}$ and $\mathrm{Te}_{4,5}$ edges. This confirmed that at least $80 \%$ of all the observed filled SWNTs were filled with microstructures consistent with our modeling protocols. TEM and image simulations were performed with the SimulaTEM multislice image simulation code using an accelerating voltage of $80 \mathrm{kV}$, a coefficient (Cs) of $0.001 \mathrm{~mm}$, and a value of defocus of $7 \mathrm{~nm} .{ }^{50} \mathrm{ADF}$ STEM simulations were performed using clTEM, an open source GPU accelerated multislice program developed by M. A. Dyson. ${ }^{51}$ The simulation parameters were matched to the microscope aberrations as measured by the CEOS control software and also duplicating the imaging conditions stated above for either the ARM200F electron microscope operating in STEM mode at $80 \mathrm{kV}$ or the Nion UltraSTEM100 dedicated STEM operated at $60 \mathrm{kV}$. Thermal diffuse scattering was modeled using the frozen phonon method with 15 configurations.

$\boldsymbol{A} \boldsymbol{b}$ Initio Computations. We employ density-functional theory and density-functional perturbation theory with a plane-wave basis set as implemented in CASTEP-17.1 $1^{52}$ and Quantum Espresso-6.1. ${ }^{53} \mathrm{We}$ use a wave functions basis set with a cutoff energy of $400 \mathrm{eV}$; the maximum distance in the k-point grid in reciprocal space is $1 \AA^{-1}$. In the AIRSS search the total energy is converged within $1 \times 10^{-6} \mathrm{eV}$ tolerance, and the maximum module of forces for geometry optimization is $0.05 \mathrm{eV} / \AA$. Details on employed pseudopotentials, exchange-correlation functional and convergence parameters for phonon calculations, band structure, electron-phonon coupling, and Wannierisation of wave functions are described in the Supporting Information.

\section{ASSOCIATED CONTENT}

S Supporting Information

The Supporting Information is available free of charge on the ACS Publications website at DOI: 10.1021/acsnano.8b02261.

More information about the structural models, electronic structures, mechanical stability, and charge analysis; details of conducted calculations and exploited methods for calculation of transport properties ${ }^{52-57}$ (PDF)

\section{AUTHOR INFORMATION}

\section{Corresponding Authors}

*E-mail: A.Vasylenko@warwick.ac.uk.

*E-mail: J.Sloan@warwick.ac.uk.

*E-mail: D.Quigley@warwick.ac.uk.

ORCID

Andrij Vasylenko: 0000-0002-6933-0628

Paulo V. C. Medeiros: 0000-0002-7803-9058

Quentin M. Ramasse: 0000-0001-7466-2283

Andrew J. Morris: 0000-0001-7453-5698

\section{Notes}

The authors declare no competing financial interest.

Data used in this work are available via the Warwick data repository at wrap.warwick.ac.uk/100297.

\section{ACKNOWLEDGMENTS}

A.V., P.V.C.M., A.J.M., J.S., and D.Q. thank the UK Engineering and Physical Sciences Research Council (EPSRC) for funding through grant numbers EP/M010643/1 and EP/M011925/1. J.M.W. acknowledges financial support from the EPSRC Centre for Doctoral Training in Computational Methods for Materials Science under grant EP/L015552/1. S.M. and J.S. are further indebted to support from EP/I033394/1. The SuperSTEM Laboratory is the U.K. National Facility for AberrationCorrected STEM, supported by the EPSRC. This work used the ARCHER UK National Supercomputing Service (www. archer.ac.uk).

\section{REFERENCES}

(1) Heremans, J. P.; Dresselhaus, M. S.; Bell, L. E.; Morelli, D. T. When Thermoelectrics Reached the Nanoscale. Nat. Nanotechnol. 2013, 8, 471-473.

(2) Ferry, D. K. Nanowires in Nanoelectronics. Science 2008, 319, $579-580$.

(3) Toberer, E. S.; Zevalkink, A.; Snyder, G. J. Phonon Engineering Through Crystal Chemistry. J. Mater. Chem. 2011, 21, 15843-15852.

(4) Zhou, M.; Li, J.-F.; Kita, T. Nanostructured $\mathrm{AgPb}_{m} \mathrm{SbTe}_{m+2}$ System Bulk Materials with Enhanced Thermoelectric Performance. J. Am. Chem. Soc. 2008, 130, 4527-4532.

(5) Biswas, K.; He, J.; Blum, I. D.; Wu, C.-I.; Hogan, T. P.; Seidman, D. N.; Dravid, V. P.; Kanatzidis, M. G. High-Performance Bulk Thermoelectrics with All-Scale Hierarchical Architectures. Nature 2012, 489, 414-418.

(6) Heremans, J. P.; Jovovic, V.; Toberer, E. S.; Saramat, A.; Kurosaki, K.; Charoenphakdee, A.; Yamanaka, S.; Snyder, G. J. 
Enhancement of Thermoelectric Efficiency in PbTe by Distortion of the Electronic Density of States. Science 2008, 321, 554-557.

(7) Pei, Y.; Shi, X.; LaLonde, A.; Wang, H.; Chen, L.; Snyder, G. J. Convergence of Electronic Bands for High Performance Bulk Thermoelectrics. Nature 2011, 473, 66-69.

(8) Liu, W.; Tan, X.; Yin, K.; Liu, H.; Tang, X.; Shi, J.; Zhang, Q.; Uher, C. Convergence of Conduction Bands as a Means of Enhancing Thermoelectric Performance of $n$-Type $\mathrm{Mg}_{2} \mathrm{Si}_{1}-x \mathrm{Sn}_{x}$ Solid Solutions. Phys. Rev. Lett. 2012, 108, 166601.

(9) Zhao, L.-D.; Tan, G.; Hao, S.; He, J.; Pei, Y.; Chi, H.; Wang, H.; Gong, S.; Xu, H.; Dravid, V. P.; Uher, C.; Snyder, G. J.; Wolverton, C.; Kanatzidis, M. G. Ultrahigh Power Factor and Thermoelectric Performance in Hole-Doped Single-Crystal SnSe. Science 2016, 351, 141-144.

(10) Fu, C.; Bai, S.; Liu, Y.; Tang, Y.; Chen, L.; Zhao, X.; Zhu, T. Realizing High Figure of Merit in Heavy-Band P-Type Half-Heusler Thermoelectric Materials. Nat. Commun. 2015, 6, 8144.

(11) Shi, X.; Yang, J.; Salvador, J. R.; Chi, M.; Cho, J. Y.; Wang, H.; Bai, S.; Yang, J.; Zhang, W.; Chen, L. Multiple-Filled Skutterudites: High Thermoelectric Figure of Merit Through Separately Optimizing Electrical and Thermal Transports. J. Am. Chem. Soc. 2011, 133, 7837-7846.

(12) Pei, Y.; Wang, H.; Snyder, G. J. Thermoelectric Materials: Band Engineering of Thermoelectric Materials. Adv. Mater. 2012, 24, 61246124.

(13) Wang, Z.; Alaniz, J. E.; Jang, W.; Garay, J. E.; Dames, C. Thermal Conductivity of Nanocrystalline Silicon: Importance of Grain Size and Frequency-Dependent Mean Free Paths. Nano Lett. 2011, 11, 2206-2213.

(14) Hochbaum, A. I.; Chen, R.; Delgado, R. D.; Liang, W.; Garnett, E. C.; Najarian, M.; Majumdar, A.; Yang, P. Enhanced Thermoelectric Performance of Rough Silicon Nanowires. Nature 2008, 451, 163167.

(15) Hicks, L. D.; Dresselhaus, M. S. Effect of Quantum-Well Structures on the Thermoelectric Figure of Merit. Phys. Rev. B: Condens. Matter Mater. Phys. 1993, 47, 12727-12731.

(16) Tan, G.; Zhao, L.-D.; Shi, F.; Doak, J. W.; Lo, S.-H.; Sun, H.; Wolverton, C.; Dravid, V. P.; Uher, C.; Kanatzidis, M. G. High Thermoelectric Performance of P-Type SnTe via a Synergistic Band Engineering and Nanostructuring Approach. J. Am. Chem. Soc. 2014, 136, 7006-7017.

(17) Tan, G.; Shi, F.; Hao, S.; Chi, H.; Bailey, T. P.; Zhao, L.-D.; Uher, C.; Wolverton, C.; Dravid, V. P.; Kanatzidis, M. G. Valence Band Modification and High Thermoelectric Performance in SnTe Heavily Alloyed with MnTe. J. Am. Chem. Soc. 2015, 137, 1150711516.

(18) Zheng, L.; Li, W.; Lin, S.; Li, J.; Chen, Z.; Pei, Y. Interstitial Defects Improving Thermoelectric SnTe in Addition to Band Convergence. ACS Energy Lett. 2017, 2, 563-568.

(19) Wuttig, M.; Yamada, N. Phase-Change Materials for Rewriteable Data Storage. Nat. Mater. 2007, 6, 824-832.

(20) Lencer, D.; Salinga, M.; Grabowski, B.; Hickel, T.; Neugebauer, J.; Wuttig, M. A Map for Phase-Change Materials. Nat. Mater. 2008, 7, 972-977.

(21) Spencer, J. H.; Nesbitt, J. M.; Trewhitt, H.; Kashtiban, R. J.; Bell, G.; Ivanov, V. G.; Faulques, E.; Sloan, J.; Smith, D. C. Raman Spectroscopy of Optical Transitions and Vibrational Energies of $1 \mathrm{~nm}$ $\mathrm{HgTe}$ Extreme Nanowires within Single Walled Carbon Nanotubes. ACS Nano 2014, 8, 9044-9052.

(22) Carter, R.; Suyetin, M.; Lister, S.; Dyson, A.; Trewhitt, H.; Goel, S.; Liu, Z.; Suenaga, K.; Giusca, C.; Kashtiban, R.; Hutchison, J.; Dore, J.; Bell, G.; Bichoutskaia, E.; Sloan, J. Band Gap Expansion, Shear Inversion Phase Change Behaviour and Low-Voltage Induced Crystal Oscillation in Low-Dimensional Tin Selenide Crystals. Dalton Trans. 2014, 43, 7391-7399.

(23) Sloan, J.; Grosvenor, S. J.; Friedrichs, S.; Kirkland, A. I.; Hutchison, J. L.; Green, M. L. H. A One-Dimensional BaI ${ }_{2}$ Chain With Five- and Six-Coordination, Formed within a Single-Walled Carbon Nanotube. Angew. Chem., Int. Ed. 2002, 41, 1156-1159.
(24) Medeiros, P. V. C.; Marks, S.; Wynn, J. M.; Vasylenko, A.; Ramasse, Q. M.; Quigley, D.; Sloan, J.; Morris, A. J. Single-Atom Scale Structural Selectivity in Te Nanowires Encapsulated Inside Ultranarrow, Single-Walled Carbon Nanotubes. ACS Nano 2017, 11, 61786185.

(25) Philp, E.; Sloan, J.; Kirkland, A. I.; Meyer, R. R.; Friedrichs, S.; Hutchison, J. L.; Green, M. L. H. An Encapsulated Helical OneDimensional Cobalt Iodide Nanostructure. Nat. Mater. 2003, 2, 788791.

(26) Senga, R.; Komsa, H.-P.; Liu, Z.; Hirose-Takai, K.; Krasheninnikov, A. V.; Suenaga, K. Atomic Structure and Dynamic Behaviour of Truly One-Dimensional Ionic Chains Inside Carbon Nanotubes. Nat. Mater. 2014, 13, 1050-1054.

(27) Wynn, J. M.; Medeiros, P. V. C.; Vasylenko, A.; Sloan, J.; Quigley, D.; Morris, A. J. Phase Diagram of Germanium Telluride Encapsulated in Carbon Nanotubes From First-Principles Searches. Phys. Rev. Mater. 2017, 1, 073001.

(28) Nikolaev, P.; Thess, A.; Rinzler, A. G.; Colbert, D. T.; Smalley, R. E. Diameter Doubling of Single-Wall Nanotubes. Chem. Phys. Lett. 1997, 266, 422-426.

(29) Smith, B. W.; Monthioux, M.; Luzzi, D. E. Encapsulated $\mathrm{C}_{60}$ in Carbon Nanotubes. Nature 1998, 396, 323-324.

(30) Sloan, J.; Kirkland, A. I.; Hutchison, J. L.; Green, M. L. H. Integral Atomic Layer Architectures of 1D Crystals Inserted into Single Walled Carbon Nanotubes. Chem. Commun. 2002, 0, 13191332.

(31) Kharlamova, M. V. Advances in Tailoring the Electronic Properties of Single-Walled Carbon Nanotubes. Prog. Mater. Sci. 2016, $77,125-211$.

(32) Lee, C. Y.; Choi, W.; Han, J.-H.; Strano, M. S. Science 2010, 329, $1320-1324$

(33) Chamberlain, T. W.; Biskupek, J.; Rance, G. A.; Chuvilin, A.; Alexander, T. J.; Bichoutskaia, E.; Kaiser, U.; Khlobystov, A. N. Size, Structure, and Helical Twist of Graphene Nanoribbons Controlled by Confinement in Carbon Nanotubes. ACS Nano 2012, 6, 3943-3953.

(34) Zhang, Y.; Wang, F.-C.; Zhao, Y.-P. Negative Differential Resistance Behavior of Silicon Monatomic Chain Encapsulated in Carbon Nanotubes. Comput. Mater. Sci. 2012, 62, 87-92.

(35) Safdar, M.; Wang, Q.; Mirza, M.; Wang, Z.; Xu, K.; He, J. Topological Surface Transport Properties of Single-Crystalline SnTe Nanowire. Nano Lett. 2013, 13, 5344-5349.

(36) Herring, C. Theory of the Thermoelectric Power of Semiconductors. Phys. Rev. 1954, 96, 1163.

(37) Pickard, C. J.; Needs, R. J. High-Pressure Phases of Silane. Phys. Rev. Lett. 2006, 97, 045504.

(38) Harman, P. M. Scientific Letters and Papers of J. C. Maxwell; Cambridge University Press: Cambridge, 2002; Vol. 3; pp 1874-1879.

(39) Morris, A. J.; Grey, C. P.; Needs, R. J.; Pickard, C. J. Energetics of Hydrogen/lithium Complexes in Silicon Analyzed Using the Maxwell Construction. Phys. Rev. B: Condens. Matter Mater. Phys. 2011, $84,224106$.

(40) See Supporting Information for the details of calculations of transport properties in the SnTe nanowires.

(41) Tan, G.; Zhao, L.-D.; Shi, F.; Doak, J. W.; Lo, S.-H.; Sun, H.; Wolverton, C.; Dravid, V. P.; Uher, C.; Kanatzidis, M. G. High Thermoelectric Performance of P-Type SnTe via a Synergistic Band Engineering and Nanostructuring Approach. J. Am. Chem. Soc. 2014, 136, 7006-7017.

(42) Kosynkin, D. V.; Higginbotham, A. L.; Sinitskii, A.; Lomeda, J. R.; Dimiev, A.; Price, B. K.; Tour, J. M. Longitudinal Unzipping of Carbon Nanotubes to Form Graphene Nanoribbons. Nature 2009, 458, 872-876.

(43) Liu, Q.; Fujigaya, T.; Nakashima, N. Graphene Unrolled From 'cup-Stacked' Carbon Nanotubes. Carbon 2012, 50, 5421-5428.

(44) Viculis, L. M.; Mack, J. J.; Kaner, R. B. A Chemical Route to Carbon Nanoscrolls. Science 2003, 299, 1361-1361.

(45) Yin, H.; Cai, K. Unwinding of a Carbon Nanoscroll Due to High Speed Rotation. AIP Adv. 2015, 5, 107202. 
(46) Vasylenko, A.; Wynn, J.; Medeiros, P. V. C.; Morris, A. J.; Sloan, J.; Quigley, D. Encapsulated Nanowires: Boosting Electronic Transport in Carbon Nanotubes. Phys. Rev. B: Condens. Matter Mater. Phys. 2017, 95, 121408R.

(47) Lindsay, L.; Broido, D. A.; Mingo, N. Lattice Thermal Conductivity of Single-Walled Carbon Nanotubes: Beyond the Relaxation Time Approximation and Phonon-Phonon Scattering Selection Rules. Phys. Rev. B: Condens. Matter Mater. Phys. 2009, 80, 125407.

(48) Kodama, T.; Ohnishi, M.; Park, W.; Shiga, T.; Park, J.; Shimada, T.; Shinohara, H.; Shiomi, J.; Goodson, K. E. Modulation of Thermal and Thermoelectric Transport in Individual Carbon Nanotubes By Fullerene Encapsulation. Nat. Mater. 2017, 16, 892.

(49) Choi, J.; Jung, Y.; Yang, S. J.; Oh, J. Y.; Oh, J.; Jo, K.; Son, J. G.; Moon, S. E.; Park, C. R; Kim, H. Flexible and Robust Thermoelectric Generators Based on All-Carbon Nanotube Yarn without Metal Electrodes. ACS Nano 2017, 11, 7608-7614.

(50) Gomez-Rodriguez, A.; Beltran-del Rio, L. M.; Herrera-Becerra, R. SIMULATEM: Multislice Simulations for General Objects. Ultramicroscopy 2010, 110, 95-104.

(51) Dyson, M. A. clTEM: OpenCLTEM/STEMsimulationcode, URL: http://github.com/ADyson/clTEM, 2014.

(52) Clark, S. J.; Segall, M. D.; Pickard, C. J.; Hasnip, P. J.; Probert, M. I. J.; Refson, K.; Payne, M. C. First Principles Methods Using CASTEP. Z. Kristallogr. - Cryst. Mater. 2009, 220, 567-570.

(53) Giannozzi, P.; Baroni, S.; Bonini, N.; Calandra, M.; Car, R.; Cavazzoni, C.; Ceresoli, D.; Chiarotti, G. L.; Cococcioni, M.; Dabo, I.; Corso, A. D.; Gironcoli, S. d.; Fabris, S.; Fratesi, G.; Gebauer, R.; Gerstmann, U.; Gougoussis, C.; Kokalj, A.; Lazzeri, M.; Martin-Samos, L.; et al. QUANTUM ESPRESSO: a Modular and Open-Source Software Project for Quantum Simulations of Materials. J. Phys.: Condens. Matter 2009, 21, 395502.

(54) Marzari, N.; Mostofi, A. A.; Yates, J. R.; Souza, I.; Vanderbilt, D. Maximally Localized Wannier Functions: Theory and Applications. Rev. Mod. Phys. 2012, 84, 1419-1475.

(55) Ponce, S.; Margine, E. R.; Verdi, C.; Giustino, F. EPW: Electron-Phonon Coupling, Transport and Superconducting Properties Using Maximally Localized Wannier Functions. Comput. Phys. Commun. 2016, 209, 116-133.

(56) Li, W.; Carrete, J.; Katcho, N. A.; Mingo, N. SHENGBTE: A Solver of the Boltzmann Transport Equation for Phonons. Comput. Phys. Commun. 2014, 185, 1747-1758.

(57) Pizzi, G.; Volja, D.; Kozinsky, B.; Fornari, M.; Marzari, N. BOLTZWANN: A Code for the Evaluation of Thermoelectric and Electronic Transport Properties with a Maximally-Localized Wannier Functions Basis. Comput. Phys. Commun. 2014, 185, 422-429. 